\title{
Research on the Specific Physical Quality Evaluation System of Junior Elite Sprinter
}

\author{
Dengfeng Zhang \\ Shandong Sport University \\ Rizhao, China 276826
}

\author{
Yunxia Li \\ Shandong Sport University \\ Rizhao, China 276826
}

\author{
Xinkai Zhang \\ Shandong Sport University \\ Rizhao, China 276826
}

\begin{abstract}
The special physical quality is the core factor to determine sprint performance, but in order to control the special physical training process quantitatively and develop the physical quality training to the established goal directionally, the precondition is to establish a specific physical quality evaluation system which is closely related to the special characteristics and sports performance on the basis of the project characteristics. This study takes 40 young athletes of Shandong Sports School as an example, through expert evaluation, correlation analysis, factor analysis and other means to construct the special physical quality evaluation system for the young elite sprint athletes, providing reference for athlete selection and training. The result shows that the index of special physical quality of youth elite sprinters can be mainly classified as specific power factor and specific speed factor. And the core training program of the special physical quality for the youth elite sprinters is 30-meter dash and 60meter dash.
\end{abstract}

Keywords-sprinter; specific physical quality; evaluation system

\section{INTRODUCTION}

The training of modern track and field events has reached a fairly high level. Especially in these years, with the development of sport training science, some new training concepts and methods have been involving in this training, which greatly improves the scientific degree of the sport training. Among them, cybernetics in contemporary system science is the theoretical basis of sprint training control. In 1970, some European and American countries and the former Soviet Union implemented the basic theory of cybernetics to optimize sports training, and they got remarkable effect, then, "Model Training Theory" was gave birth by them. The so-called model training is based on the systematic analysis of the actual situation of athletes and the prediction of their development potential. And under the guidance of the goal, the training direction and the task are identified according to the actual situation of the athletes and reasonable training skills. By the way, we can accomplish the systematic monitoring to the athletes and arrange training orderly. The establishment of the model is a systematic project, the premise of the project is the systematic collection and measurement of various factors that affect sport performance. Later, analyzing the influence of these factors in achievement and clarifying the relationship between different factors, establishing evaluation indexes of different impact factors and scientific evaluation system. Meanwhile, we should select materials and training on the basis of this model. Among the factors that affect sprinters performance, special physical fitness is the basis and the key, at the same time, it is also the vital basis for evaluation the competitive level of young sprinters combining with the characters of sprint event, and construction the evaluation system of the special physical qualities of outstanding young sprinters. The importance of promoting the development of sprint events cannot be doubted. Unfortunately, the current research on the evaluation of special physical quality of adult elite sprinters is still very weak. The lack of targeted evaluation system of special physical quality seriously restricts the development of sprint events.

\section{RESEARCH OBJECT AND METHOD}

\section{A. Research Object}

Forty talent adult sprinters in Shandong Sports University are selected, including twenty women athletes and twenty men athletes. The average age is seventeen years old, and the average training period is four to six years. The sprint grade is all in the second grade or above.

\section{B. Research Methods}

1) Literature method: In order to understand the special features of sprint we should consult sport material election, sport training and track and field training related books. Through the Chinese Web to retrieval of journals, papers and academic reports on the evaluation system of physical fitness for sports training projects, etc. and getting the information about current situation of evaluation system of physical fitness in different sports event, and to provide support for the research. 
2) Expert interview method: Visiting some experts (two professors, four associate professors), first-line coaches (three national coaches, two senior coaches and two intermediate coaches) and referees, who have been engaged in the teaching of sprint for a long time, to listen to the experts' understanding of the characteristics of young sprinter's special physical fitness, and index of extraordinary physical quality.

3) Questionnaire survey method: On the basis of expert interviews and literature research, the primary indicators are screened, supplemented and enhanced to determine the content of the questionnaire and design the expert questionnaire. Two rounds of expert questionnaire were distributed to16 track and field coached in Shandong Sport University and College of physical Education of Shandong Normal University. The two questionnaires were all recovered and valid.

4) Measurement method: According to the results of two questionnaires, Likert five-point scoring method is used to assign marks to 40 excellent young sprinters in Shandong Sports University. The average weighted score of different evaluation indexes is calculated the weighted average score of special physical qualities of 40 young elite sprinters. The primary index with scores (more than 4 or equal to 4 ) is took as the measurement index. It include 14 index: $30 \mathrm{~m}(\mathrm{~s})$, 60 (s), 100m (m), 150m (s), 200m (s), 300m (s),400m (s), 1 min Sit-up ('un) ,1 min Chin-up (once), Jumping jack (m), Squat (kg), 10s High leg lifting ('un), and Sit forward (cm).

The special quality test includes the following four steps.

- Identify testers: identify surveyors and their division of labor, train and forecast each tester to meet the condition needed for large-scale measurements and improve the accuracy of the result.

- Texting details of composing: Including text of equipment, conditions, progress methods and notes. The proper use and strict rules of texting details can contribute to improve the degree of standardization and objectivity in text.

- Determine the test time and place: Minimize the interference to training and competition caused by the test;

- To carry out the test of indicators: Carrying out special quality indicators test according to strict accordance of the preparation of physical quality measurement and evaluation methods, procedures in the National Institute of Physical Education Teaching Materials Committee, "Sports Measurement and evaluation".

5) Mathematical statistics: Statistics processing software SPSS 19.0 was used to deal with statistical data, including routine carding statistics, Pearson correlation analysis and factor analyzing. And the principal component method in factor analysis is used to construct the evaluation index model of special physical fitness of adolescent elite sprinters, and the factor, as we can see--- $\geq 70 \%$, of the cumulative contribution rate is chosen as the main factor of evaluating the excellent system.

\section{FOUNDING AND ANALYSING}

The construction process of the special physical quality evaluation model of competitive sports consists of three parts: primary selection of index, index check and evaluation system construction. First of all, through the research of same-events group project in special quality to comb to primary evaluation index, and then adopt the method of expert evaluation, the correlation analysis of index check, to construct the evaluation model. In primary stage, there are three main aspects of the source of the indicators: One is a special training coaches and experts with rich experience in the recommended indexes; the second is about the indicators involved in dash special training and special physical quality theory's results. This part is mainly from the www.cnki.net. Third, the indicators involved in the theoretical results of the special physical quality in seem-event group (physical strength leading type of rapid strength) project.

\section{A. Primary Selection of Special Physical Fitness Indicators for Outstanding Young Sprinters}

Based on the analysis of the sprint special physical characteristics, in order to make a better comprehensive screening to reflect the accurate and reasonable special physical quality index in all aspects of young excellent sprinters, following the scientific, traceable, feasible, and comprehensive principle, the study of the retrieved results can reflect the group project. Summarizing the major quality indicators, and using expert evaluation method to evaluate the proposed indicators, and to select the factors as primary quality indicators which more than 4 of likert score from the above 21 indicators (including crouch, standing long jump, 4 x $10 \mathrm{~m}$ reverse layup, bench press; $10 \mathrm{~s}$ high leg lifts in place , crook proneness, bench press; $3 \mathrm{~m}$ and $60 \mathrm{~m}, 100 \mathrm{~m}$ and $150 \mathrm{~m}, 200 \mathrm{~m}$ and $300 \mathrm{~m}, 400 \mathrm{~m}, 80 \mathrm{~m}$ and $1000 \mathrm{~m} ; 30 \mathrm{sV}$-up 、 30stwo head up, push-ups, single leg squat, and stand side flip a solid sphere;1min sit-up;1min pull-ups) Including crouch, standing long jump, $1 \mathrm{~min}$ sit-ups, $1 \mathrm{~min}$ pull-ups, $10 \mathrm{~s}$ high leg lifts in place, crook proneness, $30 \mathrm{~m}$ and $60 \mathrm{~m}, 100 \mathrm{~m}$, $150 \mathrm{~m}$ and $200 \mathrm{~m}, 300 \mathrm{~m}, 400 \mathrm{~m}$, these indicators were highly recognized by the experts, which is able to reflect the excellent young sprinters special physical quality level of the index factors from different aspects.

\section{B. The Check of Special Physical Quality in Young Sprinters}

The special physical quality of 40 excellent young sprinters in Shandong sports school was tested According to the standard test procedure, and the test results were imported into statistical software SPSS 19.0.

Due to complexity, diversity and other significant characteristics of special physical quality, the particular special physical quality test method is not the only one, and different indicators are measurable for quality level. These different measurements can response to a certain extent to 
measure quality. However, some indicators may be caused measurement work because of too strong correlation. It not only increased the quantity and difficulty of measurement in daily training, also caused the different levels of labor and material resources waste. Then this needs through the correlation analysis to eliminate possible repetitive measurement indicators, and to ensure the mutual independence of special quality indexes.

\section{The Special Physical Quality of Young Excellent Sprinters' Factor Analysis}

The factor analysis was mainly conducted through the idea of dimension reduction to take multiple index decomposition and to discover the common factors which implied in there. The common factor cannot be directly observed, it hidden behind the multiple indexes. What's more, the main purpose of the factor analysis is to find common factor, and to make the original variables which are complex and have overlapping information into few unrelated factors as few as possible indicators was used to reflect the most of the original information.

According to the thought of factor analysis, before factor analysis, the data should be verified through KMO test and Bartlett sphericity test to see if it is suitable for factor analysis. The results of the special physical quality test of excellent young sprinters were analyzed by the four checked indexes.

Statistics show that, through the factor analysis to get the two factors(special power factor and special speed) of young excellent sprinters special physical quality and the correlation between these two factors is low ( $\mathrm{P}>0.05)$, shows that these two kinds of factors can reflect the special physical characteristics of young excellent sprinters from different extent, which also means these two kinds of factors' information that is reflected in special characteristics of adolescent excellent sprinters is independent. It also shows that through factor analysis to build a classification of the excellent young sprinter's quality indexes is reasonable, and also possible, which confirms the scientific nature of the income factor.

\section{Weight of Special Physical Quality Indicators for Outstanding Sprinters of Teenagers}

The results of the analysis of the special quality index factors of 40 excellent young sprinters in Shandong sports school are shown that the contribution rate of variance of different indicators in different factors is significantly different. That is to say, there are many differences in the special quality characteristics of excellent sprinters among teenagers reflected by different indicators.

The results show that among the special power factors, the weight of the selected half squat and standing long jump factors is the largest, while the weight of each factor index in the speed factor is relatively small, which shows that the excellent sprinters should have good strength quality and directly indicates that the naming method of factors adopted in this study is reasonable. Among the special speed factors, $30 \mathrm{~m}$ and $60 \mathrm{~m}$ have the largest weight, due to the high intensity of modern sprinting competitions, any slight technical difference may lead to a significant change in the results of the competitions. These changes have raised the bar for sprinters. There are no defects in every stage from starting, accelerating, and running to the final sprint. Speed is the fundamental factor that determines the result of a match. Athletes are required to have good speed quality to adapt to the increasingly fierce competition. From the comparison of final weight, it can be seen that the weight of $30 \mathrm{~m}$ running is the largest, and then is the $60 \mathrm{~m}$ running, which indicates that strengthening speed quality training is the focus of special physical quality training. In addition, we should give more training to sprinters' strength (especially the explosive power of lower limbs).

\section{CONCLUSION}

Through literature retrieval and expert interview, 21 evaluation indexes can reflect the special physical quality of excellent sprinters of teenagers. It uses the statistics analysis to check the selected indicators, and a total of 4 indicators including squats, standing long jump, $30 \mathrm{~m}$ and $60 \mathrm{~m}$ were selected. The special physical quality index model of adolescent excellent sprinters is preliminarily constructed.

We use factor analysis for dimension check indexes, the special physical quality of adolescents excellent sprinters are classified as the special power factor, the speed factor, this two main ingredients. In terms of factor scores, special speed factor $>$ special power factor. From the final weight of the special quality index, it can be seen that the weight of the two special speed factors of $30 \mathrm{~m}$ and $60 \mathrm{~m}$ runs is relatively large, which should be the core content of the special physical quality training for the excellent sprinters of teenagers.

\section{REFERENCES}

[1] Qiao Decai, Zhang Yunkun, Deng Shu-xun. Research progress and application of sports human science [M]. Beijing: Peoples Sports Publishing House, 2008:232.

[2] Yin Junzhu. Track and field training process control theory [M]. Beijing: Beijing sport university press,2005:24.

[3] Si Huke, Zhao Bingjun, Zhu Kun, et al. Re understanding of the relationship between special technology and physical quality in track and field training [J]. Sports research and education,2004,24(6):49-51

[4] Ma Liya, Xu Shiyan. A study on the special body quality index system and evaluation criteria of China's excellent female high jump athletes [J]. Journal of Tianjin sports university,2010(3):270-274.

[5] Wang Kai, li Xiaoya, He Jiang-chuan, Yang Fang. Regression analysis of competitive ability characteristics and winning rate factors of women tennis players $[\mathrm{J}]$. Sports research and education,2014,(2):102-106.

[6] Zhao Xiaofeng. A comprehensive evaluation study on the special quality training level of China's excellent female hammer throwers [J] Sports science and technology literature bulletin,2010,(9):78-81.

[7] Cao Jintao. Preliminary construction of indicators and evaluation criteria for special sports quality of taekwondo athletes in henan province [D]. Central China normal university,2012.

[8] Han Chunyuan, Zhao Xiaowen, Wang Weixing. Research on the construction and evaluation system of special quality indicators for excellent male water polo players in China [J]. Journal of Beijing university of sport,2012,(6):117-121. 